\title{
Author Index for Volume 23
}

\begin{tabular}{|c|c|c|c|c|}
\hline Ai, C.R. & & 303 & Guo, J. & \\
\hline Akbari, S. & & 463 & Guo, S.J. & 89 \\
\hline Anis, S. & & 33 & Guo, S.T. & \\
\hline Beddani, C. & & 501 & Hafezi, R. & \\
\hline Ben-Simon, G. & & 111 & Han, J.Z. & \\
\hline Biyogmam, G.R. & & 219 & He, Z.H. & \\
\hline Chang, Z.H. & & 361 & Herzog, J. & \\
\hline Chen, C. & & 205 & Hu, J.S. & \\
\hline Chen, H.Y. & & 23 & Hua, X.H. & \\
\hline Chen, J.K. & & 239 & Kakkar, V. & \\
\hline Chen, M. & & 167 & Karim D. & \\
\hline Chen, X.H. & & 385 & Kiani, D. & \\
\hline Crupi, M. & 293 & 519 & Kose, H. & \\
\hline de Bondt, M. & & 663 & Kurtulmaz, Y. & \\
\hline De Falco, M. & & 213 & Kuzmina, A.S. & \\
\hline de Giovanni, F. & & 181 & La Barbiera, M. & \\
\hline Engel, A.-K. & & 263 & Li, F.G. & \\
\hline Eshraghi, H. & & 97 & Li, J.C. & \\
\hline Fa, H.X. & & 443 & Li, Y.T. & \\
\hline Failla, G. & & 281 & Li, Z.W. & \\
\hline Faramarzi Salles, A. & & 423 & Lian, H.F. & \\
\hline Ferrò, C. & & 519 & Liao, J. & \\
\hline Fine, B. & & 263 & Liu, H.G. & \\
\hline Gao, J.W. & & 361 & Liu, W.D. & \\
\hline Gao, Y. & 129 & 361 & Liu, Y.J. & \\
\hline Ghezelahmad, S.K. & & 463 & Lotfi Parsa, M. & \\
\hline Goodson, J.E. & & 681 & $\mathrm{Lu}, \mathrm{M}$. & 1 , \\
\hline Gordienko, A.S. & & 481 & Lu, X.M. & \\
\hline Guo, J. & & 51 & Martusciello, M. & \\
\hline
\end{tabular}


Messirdi, W.

Moussavi, A.

Musella, C.

Mushtaq, Q.

Payrovi, Sh.

Puig, L.

Rosenberger, G.

Rump, W.

Russo, A.

Saeedi Madani, S.

Salarian, Sh.

Shabani Attar, M.

Shi, J.T.

Shi, Y.Q.

Shukla, R.P.

Širovnik, N.

Song, G.J.

Spaggiari, F.

Tang, L.M.

Telloni, A.I.

Utano, R.

Vukman, J.

Wang, K.S.

Wang, L.

Wang, Q.W.

Wang, S.H.

Wang, S.X.

Wang, X.L.

Wang, Y.J.

Wang, Y.L.

Welker, V.

$\mathrm{Wu}$, T.S.

Xiong, $\mathrm{H}$.

$\mathrm{Xu}, \mathrm{A} . \mathrm{M}$.
501

335

213

33

329

541

263

149

181

469

97

651

325

129

409

65

167

117

347

117

281

65

227

71

71

427

89

189

129

623

689

51

15

251
Xu, X.Z.

Yan, D.

663

Yan, H.Y.

137

Yang, S.L.

303

Yaraneri, E.

463

Yassemi, S.

689

You, H.

507

Yu, S.W.

167

443

105

Zarrin, M.

325

701

Zhang, Y.

531

Zhang, Z.R.

701

Zhao, X.G.

361

Zhou, X.M.

507 